\section{Simvastatin as prophylactic treatment for cerebral vasospasm}

Cerebral vasospasm is a major cause of morbidity and mortality following aneurysmal subarachnoid hemorrhage (SAH). A recent randomized clinical trial in the US has investigated the ability of simvastatin to reduce vasospasm after aneurysmal $\mathrm{SAH}$.

In this study, 39 patients who had presented to hospital within 48 hours of an SAH were randomized to simvastatin $80 \mathrm{mg}$ daily $(n=19)$ or placebo $(n=20)$ for 14 days. Daily blood samples were collected for analysis of serum markers of brain injury such as $\mathrm{S} 100 \beta$, and laboratory test results were evaluated weekly for early signs of hepatitis or myositis. The primary endpoint was vasospasm defined by a clinical impression of delayed ischemic neurological deficit not related to rebleed, infection or hydrocephalus, in the presence of one or more confirmatory radiographic tests.

The mean plasma S100 $\beta$ concentration was found to be almost fourfold lower in patients receiving simvastatin than in those receiving placebo $(P<0.01)$, and the mean von Willebrand factor concentration was nearly two fold lower in the treatment arm $(P<0.05)$. Five of the 19 simvastatin patients (26\%) showed evidence of vasospasm development, compared with 12 of the 20 patients $(60 \%)$ receiving placebo. A significant decrease was observed in the maximum mean middle cerebral artery transcranial Doppler velocity for the simvastatin group compared with placebo $(P<0.01)$. No patients developed symptoms of hepatitis or myopathy.

The authors conclude that the use of simvastatin as prophylaxis against delayed cerebral ischemia after aneurysmal SAH is safe and well tolerated, and reduces the incidence of vasospasm.

Christine Kyme

Original article Lynch JR et al. (2005) Simvastatin reduces vasospasm after aneurysmal subarachnoid hemorrhage: results of a pilot randomized clinical trial. Stroke 36: 2024-2026

\section{Ultrasound guidance for caudal epidural steroid injection}

Caudal epidural steroid injection $(E S I)$ is an established treatment for spine-related problems, although reported success rates vary, largely as a result of incorrect placement of the needle. Use of fluoroscopy improves the accuracy of needle placement, but poses a radiation hazard to the patient. A recent study suggests that ultrasound might offer a safer alternative to fluoroscopy in ESI.

In this study of patients with low back pain and sciatica, Yoon and colleagues used color Doppler ultrasonography guidance to position the needle during $\mathrm{ESI}$ and to visualize any vascular intake of the medication. Needle position was then verified using injection of a contrast dye and fluoroscopy. Under ultrasound guidance, medication was successfully injected (defined as unidirectional flow on color Doppler) into the caudal space on first placement in 47 out of 53 patients. Of the 6 failures, the needle was successfully reset in 5 , giving an overall success rate of 52 out of 53 patients. Correct placement of the needle was confirmed by fluoroscopy in 50 of the 52 successfully injected patients.

The accuracy of steroid injection into the caudal space using ultrasound guidance was comparable with that obtained using fluoroscopic guidance. The authors conclude that ultrasound is preferable to fluoroscopy for needle guidance during $\mathrm{ESI}$ as it is more convenient and does not cause a radiation hazard.

Carol Lovegrove

Original article Yoon JS et al. (2005) The feasibility of color Doppler ultrasonography for caudal epidural steroid injection. Pain 118: 210-214

\section{The FOUR score: a new scale for improved assessment of coma}

The Glasgow Coma Scale (GCS) is the most widely used method for evaluation of coma, but it has a number of shortcomings, including limited utility in intubated patients and an inability to assess brainstem reflexes. A new coma score, the Full Outline of UnResponsiveness (FOUR) has been developed by Wijdicks et al. to overcome these shortcoming and to provide further neurological details that might predict outcome in coma. Four different components are included in the FOUR score (eye, motor, brainstem and respiration), each of which has a maximal score of 4 . In contrast to the GCS, verbal response is not a component of the FOUR score, making it fully applicable in intubated patients. 